OPEN ACCESS

Edited by:

Monica Guma,

University of California, San Diego,

United States

Reviewed by:

Vincenzo Venerito,

University of Bari Aldo Moro, Italy

Trine N. Jorgensen,

Case Western Reserve University,

United States

Valentina Pucino

University of Birmingham,

United Kingdom

*Correspondence:

Ursula Fearon

fearonu@tcd.ie

Specialty section:

This article was submitted to

Autoimmune and Autoinflammatory Disorders,

a section of the journal

Frontiers in Immunology

Received: 25 February 2021 Accepted: 07 June 2021

Published: 24 June 2021

Citation:

O'Brien A, Hanlon MM, Marzaioli V, Wade SC, Flynn K, Fearon U and Veale DJ (2021) Targeting JAK-STAT

Signalling Alters PSA Synovial

Fibroblast Pro-Inflammatory and Metabolic Function.

Front. Immunol. 12:672461. doi: 10.3389/fimmu.2021.672461

\section{Targeting JAK-STAT Signalling Alters PsA Synovial Fibroblast Pro- Inflammatory and Metabolic Function}

\author{
Aisling O'Brien 1,2, Megan Mary Hanlon 1,2, Viviana Marzaioli ${ }^{1,2}$, Siobhan C. Wade ${ }^{1,2}$, \\ Keelin Flynn ${ }^{2}$, Ursula Fearon ${ }^{1,2 *}$ and Douglas J. Veale ${ }^{2}$ \\ ${ }_{1}^{1}$ Molecular Rheumatology, School of Medicine, Trinity Biomedical Sciences Institute, Trinity College Dublin, Dublin, Ireland, \\ 2 Rheumatology European League against Rheumatism (EULAR) Centre of Excellence, Centre for Arthritis \& Rheumatic \\ Diseases, St Vincent's University Hospital, University College Dublin, Dublin, Ireland
}

Objectives: Psoriatic arthritis (PSA) is a chronic inflammatory disease associated with psoriasis. Janus Kinase inhibitors (JAKi) have emerged as an encouraging class of drugs for the treatment of PsA. Here, we compare the effect of four JAKi on primary PsA synovial fibroblasts (PSAFLS) activation, metabolic function, and invasive and migratory capacity.

Methods: Primary PSAFLS were isolated and cultured with JAKi (Peficitinib, Filgotinib, Baricitinib and Upadacitinib) in the presence of Oncostatin M (OSM). pSTAT3 expression in response to OSM was quantified by Western Blot analysis. Pro-inflammatory cytokines/ chemokines were quantified by ELISA and cell migration by wound-repair scratch assays. Invasive capacity was examined using Matrigel ${ }^{\mathrm{TM}}$ invasion chambers and MMP multiplex MSD assays. PsAFLS bioenergetics was assessed using the Seahorse $X F^{e}$ Extracellular Flux Analyzer, which simultaneously quantifies two energetic pathways- glycolysis (ECAR) and oxidative phosphorylation (OCR). In parallel, inflammatory, invasive, and migratory genes were quantified by RT-PCR.

Results: OSM induces pSTAT3 expression in PSAFLS. OSM-induced secretion of MCP-1 and IL-6 was inhibited by all JAKi with Peficitinib, Baricitinib and Upadacitinib showing the greatest effect. In contrast, JAKi had no significant impact on IL-8 expression in response to OSM. PSAFLS cell invasion, migratory capacity and MMP1, 3, and 9 were suppressed following JAKi treatment, with Peficitinib showing the greatest effect. These functional effects were accompanied by a change in the cellular bioenergetic profile of PSAFLS, where JAKi significantly decreased glycolysis and the ECAR/OCR, resulting in a shift to a more quiescent phenotype, with Peficitinib demonstrating the most pronounced effect.

Conclusion: This study demonstrates that JAK/STAT signalling mediates the complex interplay between inflammation and cellular metabolism in PsA pathogenesis. This inhibition shows effective suppression of inflammatory mechanisms that drive pathogenic functions of PSAFLS, further supporting the role of JAKi as a therapeutic target for the treatment of PsA.

Keywords: psoriatic arthritis, metabolism, JAK-STAT (janus kinase-signal transducer and activators of transcription), synovial fibroblast, synovial invasion 


\section{INTRODUCTION}

Psoriatic arthritis (PsA) is a chronic disease characterised by joint destruction and associated psoriasis (PsO) (1, 2). PsA synovitis is characterised by dysfunctional angiogenesis, followed by infiltration of both innate and adaptive immune cells. This leads to proliferation and activation of synovial fibroblast cells (FLS), a major source of pro-inflammatory mediators and matrix-degrading enzymes which orchestrate the persistent infiltration of immune cells and invade adjacent cartilage and bone (1-4). Furthermore, PsAFLS can further induce angiogenesis, promoting a more dysregulated endothelial cell (EC) phenotype compared to that of rheumatoid arthritis synovial fibroblast-like cells (RAFLS) (5), a phenotype consistent with the macroscopic appearance of blood vessels in vivo $(6,7)$. Indeed, PsAFLS alter the morphology, migratory and adhesive functions of ECs, in addition to their metabolic profile (5).

Many proinflammatory cytokines have been implicated in the pathogenesis of PsA, including TNF, IL-17A and IL-12/IL-23 pathways which play a key role in promoting the inflammatory response (8-11). Recently, targeted agents developed for PsA treatment include inhibitors of the Janus-Kinase (JAK) family of receptor-associated tyrosine kinases (12). Activated JAKs recruit and activate signal transducer and activator of transcription (STATs), which in turn drives gene transcription $(13,14)$. There are four JAK isoforms: JAK1, JAK2, JAK3 and TYK2, which depending on their stimulus induce the phosphorylation of different STAT proteins. Despite ongoing clinical trials, few studies have examined the precise effect of these agents in PsA synovial tissue cell subtypes, and thus our understanding of the comparative effect by which they reduce inflammation in the PsA joint is limited. Fiocco et al. demonstrated increased expression of the JAK1/STAT3/STAT5 transcriptional network associated with joint specific T cell populations in PsA (15). Consistent with this, studies have demonstrated in PsA that Tofacitinib, a JAKi, can regulate the frequency of pathologic $\mathrm{CD} 4{ }^{+} \mathrm{CD} 11 \mathrm{a}^{+} \mathrm{CD} 45 \mathrm{RO}^{+}$ IL- $17^{+} \mathrm{T}$ cells (16), inhibit Mo-DC differentiation through NOX5 and ROS production (17), decrease the $\mathrm{T}$ cell stimulatory capability of dendritic cells through suppression of type-I-IFN signalling (18), in addition to suppression of enthesitis in a A20 $0^{\text {yelKO }}$ animal model (19). Furthermore, we and others have shown increased expression of STAT signalling components in PsA synovial-biopsies and FLS (19, 20), with tofacitinib inhibiting spontaneous release of pro-inflammatory cytokines from exvivo PsA synovial explant cultures, in addition to inhibition of PsAFLS migratory and invasive capacity (21).

Current therapeutic options for PsA are mainly monoclonal antibody drugs targeting TNF, IL-23 or IL-17 (22). The therapeutic responses to these biologic disease modifying antirheumatic drugs (bDMARDs) can vary greatly with some patients showing inadequate responses. As the JAK/STAT pathway is central in driving both pro- and anti-inflammatory signals in immune regulation, including pathways which are involved in the pathogenesis of PsA, JAKi are now of great interest as a treatment option for PsA patients $(23,24)$. Currently, Tofacitinib is the only JAKi approved for PsA, with
Peficitinib, Filgotinib, Baricitinib and Upadacitinib in clinical trials or undergoing pre-clinical evaluation (25). Upadacitinib (SELECT- PsA 2) and Filgotinib (PENGUIN 2), both JAK1 inhibitors, are currently in placebo controlled, double-blind phase III trials for the treatment of PsA patients with inadequate responses to at least 1 DMARD $(26,27)$. In addition, approximately $25 \%$ of patients with moderate-severe PsO develop PsA. Peficitinib, a pan JAKi and Baricitinib a JAK1/ 2 inhibitor have been shown to significantly improve both clinical and histological manifestations of this skin disease in phase II clinical trials $(28,29)$.

As head-to-head comparisons are difficult to perform, the aim of this study was to directly compare the effect of Peficitinib, Filgotinib, Baricitinib and Upadacitinib on PsAFLS inflammatory responses, migratory and invasive capacity, in addition to their effect on the metabolic profile of these cells.

\section{MATERIALS AND METHODS}

\section{Patient Recruitment and Arthroscopy}

PsA patients were recruited from the Rheumatology Department, St. Vincent's University Hospital. Ethics for this study was approved by the St. Vincent's University Hospital Ethics and Medical Research Committee and was performed in accordance with the Declaration of Helsinki. All patients gave fully informed written consent. PsA patients were defined according to CASPAR criteria. Baseline demographics of the PsA cohort are shown in Table 1. Arthroscopies were performed under local anaesthetic using a Wolf $2.7 \mathrm{~mm}$ needle, and synovial tissue biopsies were obtained from the site of inflammation under direct visualisation as previously described (6). Biopsies were utilised for isolation of primary PsA synovial fibroblasts (PsAFLS). Ethics approval number RS18-055.

\section{Isolation of Primary Fibroblasts}

PsA synovial biopsies were digested with $1 \mathrm{mg} / \mathrm{ml}$ collagenase type 1 (Worthington Biochemical, Freehold, NJ, USA) in RPMI1640 (Gibco-BRL, Paisley, UK) for $4 \mathrm{~h}$ at $37^{\circ} \mathrm{C}$ in humidified air with $5 \% \mathrm{CO}_{2}$. Dissociated cells were grown to confluence in

TABLE 1 | Baseline Characteristics and clinical features of PSA Patients.

\begin{tabular}{lc} 
Demographic and Clinical Data & PsA (n = 14) \\
\hline Female sex n (\%) & $10(71.4)$ \\
Age (years) & $53.8 \pm 14.3$ \\
Disease duration (Years) & $11.7 \pm 13.1$ \\
ESR (mm/h) & $25.9 \pm 28.2$ \\
CRP (mg/L) & $7.5 \pm 12.6$ \\
No. tender joints & $2 \pm 1.8$ \\
No. swollen joints & $1.1 \pm 1.2$ \\
Pain VAS & $57.5 \pm 17.8$ \\
DAS28 & $3.1 \pm 0.9$ \\
DMARDs (in last 3 months) & $36 \%$ \\
\hline
\end{tabular}

Data presented as Mean (SD). ESR, erythrocyte sedimentation rate; CRP, C-reactive protein; TJC28, tender joint count out of 28 joints; SJC28, swollen joint count out of 28 joints; VAS, visual analog scale; DAS28, disease activity score based on 28-joint count; $D M A R D$, disease-modifying antirheumatic drugs. 
RPMI-1640, 10\% FBS (Gibco-BRL), $10 \mathrm{ml}$ of $1 \mathrm{mmol} / \mathrm{l}$ HEPES (Gibco-BRL), penicillin (100 units/ml; Biosciences), streptomycin (100 units/ml; Biosciences) and fungizone (0.25 $\mu \mathrm{g} / \mathrm{ml}$; Biosciences) before passaging. Cells were used between passages $2-8$.

\section{Stimulation of PsAFLS}

PsAFLS were seeded in 6- $\left(1 \times 10^{5}\right.$ cells/well $), 48-\left(2 \times 10^{4}\right.$ cells/ well) or $96-\left(2 \times 10^{4}\right.$ cells/well $)$ well plates and allowed to attach overnight in RPMI-1640, 10\% FBS, $10 \mathrm{ml}$ of $1 \mathrm{mmol} / \mathrm{l} \mathrm{HEPES}$, penicillin, streptomycin and fungizone. Media was removed the following day and cells were serum starved by adding RPMI1640 containing $1 \% \mathrm{FBS}, 10 \mathrm{ml}$ of $1 \mathrm{mmol} / \mathrm{l}$ HEPES, penicillin, streptomycin and fungizone for a further $24 \mathrm{~h}$. PsAFLS were then pretreated with JAKi (Peficitinib (PEF), Filgotinib (FILGO), Baricitinib (BARI) (ACHEMBLOCK, CA,USA) and Upadacitinib (UPA) (Ambeed Inc, IL, USA); $5 \mu \mathrm{M}$ ) (JAKi were reconstituted in DMSO to $100 \mathrm{mM})$ or DMSO $(5 \mu \mathrm{M}$; Sigma Aldrich) (vehicle control) for $1 \mathrm{~h}$ before being stimulated with Oncostatin M (OSM) (10 ng/ml; R\&D) for $24 \mathrm{~h}$. Concentration of $5 \mu \mathrm{M}$ for all JAKi was used based on a previous study showing the dose response of all JAKi in FLS $(29,30)$. Additional experiments were performed to examine if JAKi alter secondary downstream effects of cytokines that do not signal through the JAK-STAT. Therefore, PsAFLS were plated in a $96-\left(2 \times 10^{4}\right.$ cells/well $)$ well as outlined above and stimulated with IL-1 $\beta$ (10 ng/ml; Bio-Techne LTD, UK) +/- JAKi, with a DMSO control. Additional experiments were also performed for Tofacitinib $(5 \mu \mathrm{M})$ under OSM $(10 \mathrm{ng} / \mathrm{ml})$ or IL-1 $\beta(10 \mathrm{ng} /$ $\mathrm{ml})$ stimulation.

\section{Protein Isolation and Western Blot Analysis}

To determine the effect of OSM on pSTAT3 expression, PsAFLS $\left(1 \times 10^{5}\right.$ cells/well) were seeded in 6 -well plates. Once confluent, cells were serum starved as previously described and stimulated with OSM (10 ng/ml) overnight, unstimulated (basal) PsAFLS were used as a control. Media was removed from the PsAFLS and ice-cold RIPA (Radio-Immunoprecipitation Assay) buffer (Sigma) containing $10 \mu \mathrm{g} / \mathrm{ml}$ phosphatase inhibitor cocktail and $10 \mu \mathrm{g} / \mathrm{ml}$ protease inhibitor cocktail (Sigma) was used to extract protein from the PsAFLS. Measurement of protein concentration was performed using a BCA assay (Pierce Chemical Co, Rockford, IL, USA). Protein $(3 \mu \mathrm{g})$ was resolved by SDS-PAGE (5\% stacking, 10\% resolving), resolved proteins were then transferred onto nitrocellulose membranes (Amersham Biosciences, Buckinghamshire, UK) prior to $1 \mathrm{~h}$ blocking in wash buffer containing $5 \%$ non-fat milk with gentle agitation at room temperature. Membranes were incubated with rabbit polyclonal anti-pSTAT3 (Cell-Signaling Technology, UK), diluted in $5 \%$ non-fat milk containing $0.1 \%$ Tween 20 at $4^{\circ} \mathrm{C}$ overnight with gentle agitation. $\beta$-actin (1:5000, Sigma) was used as a loading control. Following three 15 min washes, membranes were incubated with appropriate horseradish peroxidaseconjugated secondary antibodies (1:5000) for $3 \mathrm{~h}$ at room temperature. The signal was detected using SuperSignal ${ }^{\circledR}$ West
Pico Chemiluminescent Substrate (Amersham Biosciences). Band densities were imaged using the ChemiDoc MP Imaging System (Bio-Rad, USA).

\section{Enzyme-Linked Immunosorbent Assay}

Supernatants from treated PsAFLS and DMSO control $(5 \mu \mathrm{M})$ $\left(2 \times 10^{4}\right.$ cells/well) seeded in 96-well plates were harvested and levels of IL-8, IL-6, and MCP-1 measured by specific ELISA (MCP-1: eBiosciences, USA, IL-8, IL-6; DuoSet ELISA, R\&D systems, UK) according to manufacturer's protocol.

\section{mRNA Extraction and cDNA Synthesis}

To determine the effects of JAKi on specific genes in response to OSM stimulation, PsAFLS ( $1 \times 10^{5}$ cells/well) were seeded in 6well plates and stimulated as previously described. Total RNA was isolated using an RNeasy Plus mini kit (Qiagen, Germany) according to the manufacturer's specifications. The integrity of the RNA samples was assessed using a bioanalyzer (Agilent, CA, USA). Samples with a 260:280 $\mathrm{nm}$ ratio of 1.8 or above were used in subsequent experiments. Total RNA (100 ng) was reverse transcribed to cDNA using a high capacity cDNA reverse transcription kit (Applied Biosystems, Cheshire, UK) and stored at $-20^{\circ} \mathrm{C}$ until further use.

\section{RT-PCR Analysis}

Gene expression data were quantified by RT-PCR using the QuantStudio 5 Thermal Cycler (Applied Biosystem, Lewes, UK). Reaction mixtures contained $1 \mu \mathrm{l}$ of cDNA, SYBR green PCR mastermix (Applied Biosystems) and target mRNA specific primer pairs as follows: IL-6 for 5' CCCTGAGAAAGGA GACATTGTAAC 3', IL-6 rev 5'CCTCTTTGCTGCTTT CACACATG 3', IL-8 for 5' TTGGCAGCCTTCCTGATTTC 3', IL-8 rev 5' TGGCAAAACTGCACCTTCAC 3', MCP-1 for 5' GCTCGCTCAGCCAGATGCAA 3', MCP-1 rev 5' TGGTGAA GTTATAACAGCAGGTGA 3', MMP1 for 5' GCTAACAAAT ACTGGAGGTATGATG 3', MMP1 rev 5' ATTTTGGGATAA CCTGGATCCATAG 3', ICAM for 5' AACCAGAGCCAGGA GACACTG 3', ICAM rev 5' GCGCCGGAAAGCTGTAGATG 3'.

Samples lacking multiscribe reverse transcriptase formed the negative controls to ensure target-specific quantification. Data were analysed using the comparative threshold cycle $(\mathrm{Ct})$ method with normalization to the expression of RPLPO (for $5^{\prime}$ GCGTCCTCGTGGAAGTGACATCG 3', rev 5' TCAGGGATT GCCACGCAGGG 3') and HPRT1 (for 5' ATGGACAGGAC TGAACGTCTTG 3', rev 5' GGCTACAATGTGATGGCCTC 3') as endogenous controls.

\section{Cellular Bioenergetic Function Analysis}

Oxygen consumption rate (OCR) and extracellular acidification rate (ECAR), reflecting oxidative phosphorylation and glycolysis, respectively, were measured using the Seahorse-XFe96 analyser (Seahorse Biosciences, UK). PsAFLS were seeded at $12 \times 10^{3} /$ well in a 96-well cell culture XFe microplate (Seahorse Biosciences) and allowed to adhere overnight. Following this, cells were treated with JAKi/DMSO $(5 \mu \mathrm{M})$ for $1 \mathrm{~h}$ and then stimulated with OSM (10 ng/ml) for $24 \mathrm{~h}$. Additional experiments were also performed in the presence of IL-1 $\beta$ (10 ng/ml). Basal oxidative 
phosphorylation/glycolysis were calculated by the average of five baseline OCR/ECAR measurements, respectively, obtained before injection of specific metabolic inhibitors; oligomycin (ATP-synthase-inhibitor) (2 $\mu \mathrm{g} / \mathrm{ml}$; Seahorse Biosciences), trifluorocarbonylcyanide phenylhydrazone (FCCP) (mitochondrial uncoupler) (5 $\mu \mathrm{M}$; Seahorse Biosciences) and antimycin A (complex-III inhibitor) (2 $\mu \mathrm{M}$; Seahorse Biosciences) and rotenone ( $2 \mu \mathrm{M}$; Sigma Aldrich). Oligomycin was injected to evaluate both the maximal glycolytic rate and ATP synthesis, determined by subtracting the amount of respiration left after oligomycin injection from baseline OCR. FCCP was injected to evaluate the maximal respiratory capacity (average of three measurements following injection). Maximal respiratory capacity was determined by subtracting baseline OCR from FCCP-induced OCR and the respiratory reserve (baseline OCR subtracted from maximal respiratory capacity).

\section{Migration Assay}

PsAFLS $\left(2 \times 10^{4}\right.$ cells/well) were seeded in 48 -well plates for $24 \mathrm{~h}$ and serum starved as previously described. A single scratch wound was induced through the middle of each well with a sterile pipette tip and cells were subsequently treated with JAKi/ $\operatorname{DMSO}(5 \mu \mathrm{M})$ for $1 \mathrm{~h}$ followed by stimulation with OSM $(10 \mathrm{ng} / \mathrm{ml})$ for $24 \mathrm{~h}$.

PSA FLS migration across the wound margins was assessed and photographed using a phase-contrast microscope. Semiquantitative analysis of cell repopulation of the wound was assessed. Briefly, cells were fixed with $4 \%$ paraformaldehyde, stained with $0.1 \%$ crystal violet and the number of migrating cells across the time zero margin was assessed.

\section{Transwell Invasion Assay}

BioCoat Matrigel $^{\mathrm{TM}}$ Invasion Chambers (Becton Dickinson, UK) were used to assess PsAFLS invasion. Cells were seeded at $3 \times 10^{4}$ cells/well in the migration chamber on $8 \mu \mathrm{M}$ membranes precoated with matrigel. Cells were treated with JAKi/DMSO $(5 \mu \mathrm{M})$ for $1 \mathrm{~h}$ and stimulated with OSM (10 ng/ml) for $48 \mathrm{~h}$. Non-migrating cells were removed from the upper surface by gentle scrubbing. Migrating cells attached to the lower membrane were fixed with $4 \%$ paraformaldehyde and stained with $0.1 \%$ crystal violet. Cells from five random high-power fields for each well were counted to assess the average number of invading cells.

\section{MMP 3-Plex MSD Assay}

Supernatants from stimulated PsAFLS $\left(2 \times 10^{4}\right.$ cells/well) seeded in 96-well plates were harvested for MMP1, MMP3 and MMP9 analysis by MSD assay (Meso Scale Diagnostics, USA) and MMP expression was measured according to manufacturer's protocol.

\section{Statistical Analysis}

Statistical analyses were performed using Prism 8 software. Wilcoxon Signed Rank test, one-way analysis of variance (ANOVA), Friedman Test with Dunn's multiple comparison were utilised. $p$ values of less than $0.05\left({ }^{*} p<0.05\right), 0.01$ $\left.\left({ }^{* *} p<0.01\right), 0.001{ }^{* * *} p<0.001\right)$ and $\left.0.0001{ }^{* * * *} p<0.0001\right)$ were determined as statistically significant. All raw data are available on request.

\section{RESULTS}

\section{JAK Inhibitors Alter PsAFLS Secretion of Pro-Inflammatory Mediators Induced by OSM}

As OSM was utilised to active the JAK-STAT pathway, initial experiments assessed the effect of OSM on pSTAT3 expression. Figure 1A demonstrates that OSM stimulates pSTAT3 in $n=3$ separate PsAFLS. To assess the impact of JAKi, we initially determined their effect on a range of pro-inflammatory mediators. Firstly, we stimulated the PsAFLS with OSM and found that MCP-1 and IL-6 secretion (both $\mathrm{p}<0.05$ ) were significantly increased following stimulation compared to control (Figures 1B, C). IL-6 gene expression was also significantly increased $(\mathrm{p}<0.05)$, with an increasing trend observed for MCP-1 gene expression (Figures 1B, C). MCP-1 secretion was significantly reduced by Peficitinib, Upadacitinib (both $\mathrm{p}<0.001)$ and Baricitinib $(\mathrm{p}<0.05)$ (Figure 1B). Although not significant, Filgotinib also showed a strong decrease in MCP-1 secretion. In parallel, inhibition was also observed at gene level, with Baricitinib $(\mathrm{p}<0.01)$ and Upadacitinib $(\mathrm{p}<0.05)$ significantly decreasing MCP-1 mRNA expression (Figure 1B). Similarly, JAKi also reduced OSM-induced expression of IL-6 at both the protein and gene level (Figure 1C). Peficitinib $(\mathrm{p}<0.001)$ and Upadacitinib ( $\mathrm{p}<0.01)$ displayed significant inhibition of IL-6 (Figure 1C). This observation was also observed at gene level with Baricitinib $(\mathrm{p}<0.05)$ and Upadacitinib $(\mathrm{p}<0.05)$ showing significant reductions in IL-6 expression. Although not significant, Peficitinib also displayed an inhibitory capacity on IL-6 gene expression (Figure 1C). In contrast to both MCP-1 and IL-6 expression, OSM significantly reduced IL-8 expression ( $\mathrm{p}<$ 0.05) compared to control (Figure 1D). JAKi showed no significant effect on IL-8 secretion, however, there was an increasing trend observed for IL-8 mRNA expression (Figure 1D).

\section{JAK Inhibitors Reduce the OSM-Induced Shift to Glycolysis in PsAFLS}

To examine whether the inhibitory effect of JAKi on proinflammatory mediators is paralleled by a shift in metabolism, we analysed the two major energy pathways: oxidative phosphorylation (OCR) and glycolysis (ECAR) in real time, using the Seahorse XFeAnalyser. Figure 2A displays the average bioenergetic profiles for ECAR and OCR of PsAFLS before and after injections of mitochondrial inhibitors: oligomycin, carbonyl cyanide-ptrifluoromethoxyphenylhydrazone (FCCP), antimycin A and rotenone in the presence of OSM. As shown in the seahorse profiles, stimulation with OSM increased the ECAR with no effect observed for OCR (Figure 2A). Quantification demonstrated a significant increase in baseline ECAR $(\mathrm{p}<0.05)$ (Figure 2B) with no effect observed for baseline OCR (Figure 2D). Similarly, max glycolytic capacity $(\mathrm{p}<0.05)$, but not max respiratory capacity was significantly increased by OSM stimulation (Figures 2C, E). 
A
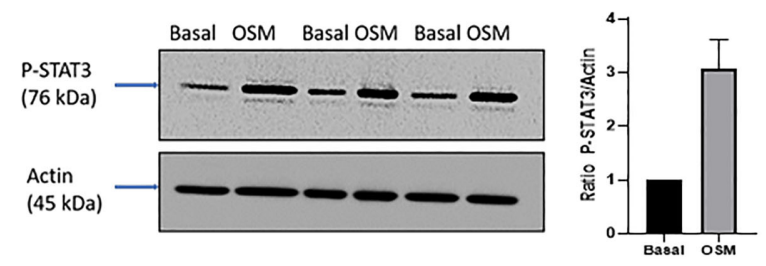

C
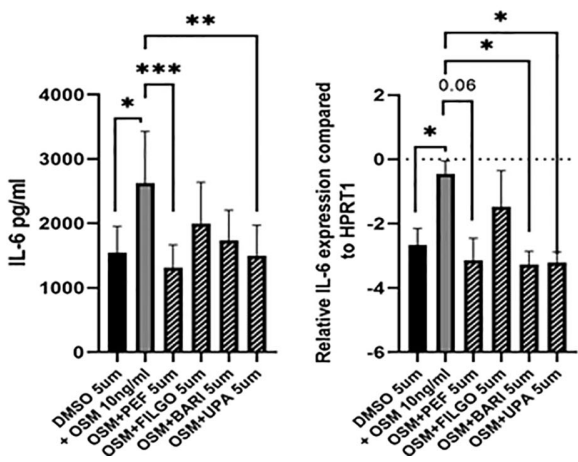

B
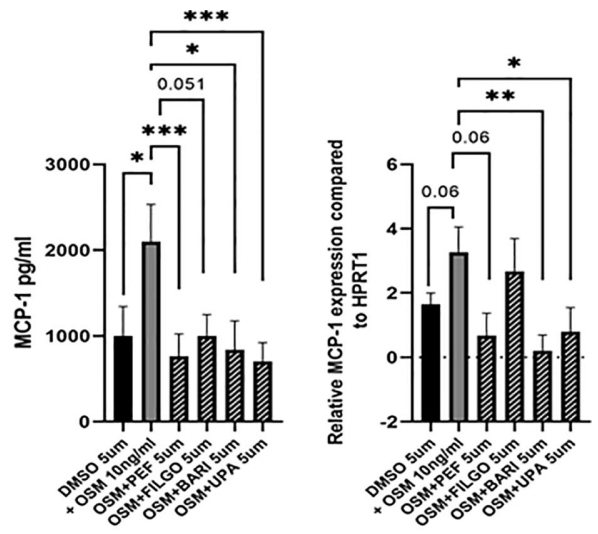

D
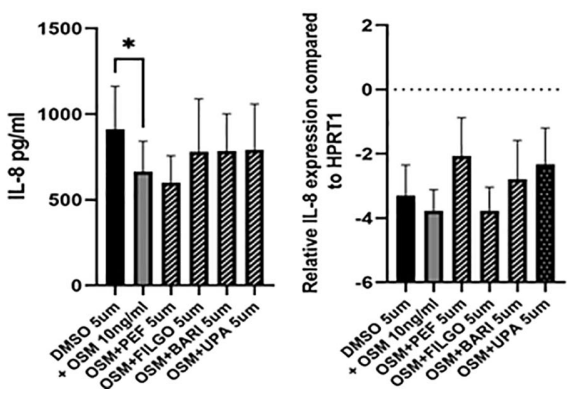

FIGURE 1 | Effect of JAKi on the OSM driven expression of pro-inflammatory mediators in PsAFLS. (A) Representative western blot images and bar graph showing pSTAT3 expression in PSAFLS stimulated with/without OSM for $24 \mathrm{~h}$. (B-D) PSAFLS were treated with JAKi $(5 \mu \mathrm{M})$ or DMSO $(5 \mu \mathrm{M})$ for $1 \mathrm{~h}$ and stimulated with $\operatorname{OSM}(10 \mathrm{ng} / \mathrm{ml})$ for $24 \mathrm{~h}$. Bar graphs demonstrating secretion by ELISA ( $n=7)$ and gene expression by real-time PCR ( $n=4-5)$ for MCP-1 (B) IL-6 (C) and IL-8 (D). Values expressed \pm SEM. ${ }^{*} p<0.05,{ }^{* *} p<0.01,{ }^{* * *} p<0.001$ significantly different from DMSO+OSM control.

Treatment with JAKi demonstrated a decrease in the ECAR bioenergetic profile for all JAKi, with minimal change observed for the OCR bioenergetic profile (Figure 2A). All JAKi reduced the OSM- induced glycolytic capacity of PsAFLS with a significant decrease in basal glycolytic capacity in response to Peficitinib ( $\mathrm{p}<$ 0.01) and Baricitinib ( $<<0.01)$ (Figure 2B), and a significant decrease in Maximal Glycolytic Capacity in response to Baricitinib ( $\mathrm{p}<0.0001$ ) (Figure 2C). No change in basal respiration (Figure 2D), or maximal respiratory capacity in response to JAKi was observed (Figure 2E). This therefore resulted in a significant decrease in the ECAR : OCR ratio, signifying a shift away from glycolytic mechanisms and towards a reliance on mitochondrial respiration. This was most significant following treatment with Peficitinib ( $\mathrm{p}<0.01)$, Baricitinib $(\mathrm{p}<0.05)$ and Upadacitinib $(\mathrm{p}<$ 0.05) (Figure 2F). Filgotinib also decreased both the glycolytic capacity and ECAR : OCR ratio, but this did not reach significance (Figure 2F). The impact of JAKi on PsAFLS bioenergetics is clearly demonstrated in the overall metabolic profile, whereby the glycolytic profiles of PsAFLS induced by OSM, shift towards a more quiescent state following treatment with JAKi (Figure 2G). To examine if JAKi alter secondary downstream effects of other cytokines that do not signal through the JAK-STAT pathway, we examined their effect on IL-1 $\beta$-induced PsAFLS (Figures 3 and 4). JAKi have no effect on IL-1 $\beta$-induced MCP-1 secretion (Figure 3A). While some inhibitory effect was observed for IL-1 $\beta$-induced IL-6 expression, this is not significant (Figure 3B). We also examined the bioenergetic profile of PsAFLS stimulated with IL-1 $\beta$ and the four JAKi (Figures 3C-F), in addition to Tofacitinib (Figures $4 \mathbf{A}-\mathbf{C}$ ). IL-1 $\beta$ increased the ECAR but not the OCR of the cells, with the JAKi's including Tofacitinib showing minimal effect on either energetic pathways (Figures 3C-F and Figures 4A-C). Tofacitinib which predominantly inhibits JAK3 and to a lesser extent JAK2 significantly inhibited both MCP-1 and IL- 6 in response to OSM (Figure 4D). Similar to the other JAKi, Tofacitinib had no effect on IL-1 $\beta$-induced MCP-1 and IL-6 secretion (Figure 4E).

\section{OSM Driven PsAFLS Invasion Is Inhibited by JAKi}

To further examine the effect of JAKi on PsAFLS pathogenic function, we examined the effect of JAKi on the invasive capacity of PsAFLS using Transwell Matrigel ${ }^{\mathrm{TM}}$ invasion chambers following stimulation with OSM. Representative images of PsAFLS invasion in unstimulated cells, OSM stimulated cells and OSM stimulated cells following treatment with JAKi are shown in Figure 5A. Quantitative analysis demonstrated the significant increase in invasive capacity following OSM stimulation $(\mathrm{p}<0.05)$ compared to unstimulated cells (Figure 5B). This was significantly impacted by treatment with all JAKi, however Peficitinib ( $\mathrm{p}<0.001)$ and Filgotinib $(\mathrm{p}<0.05)$ 

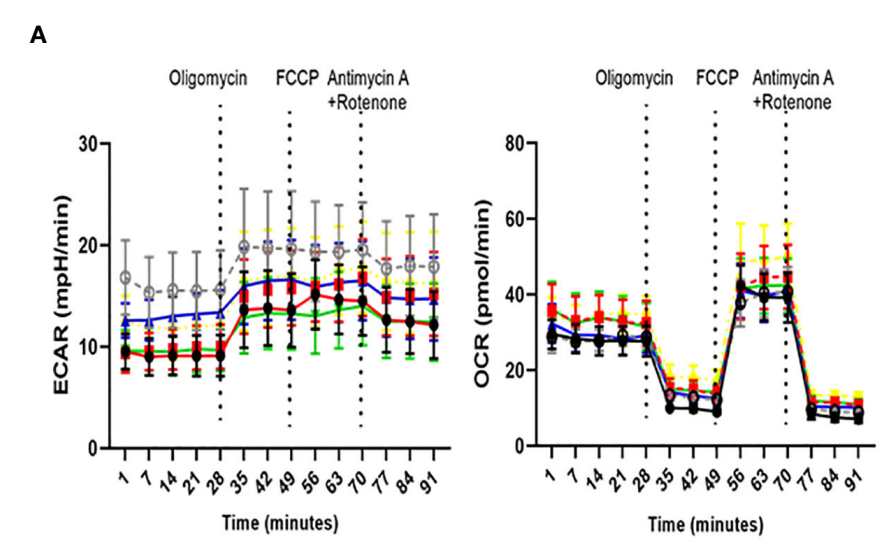

D
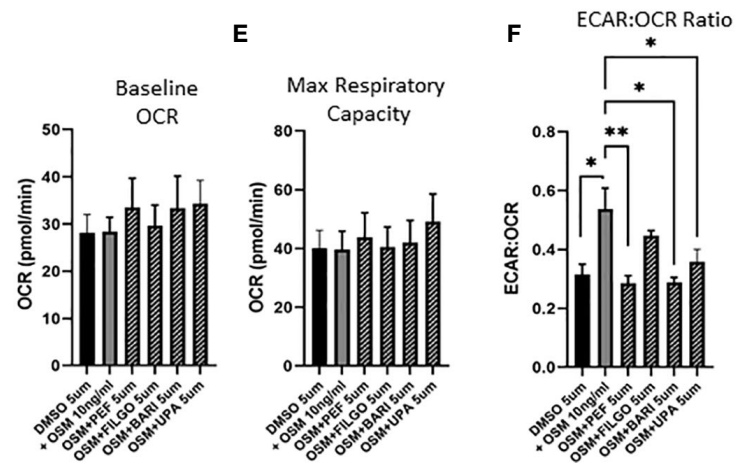

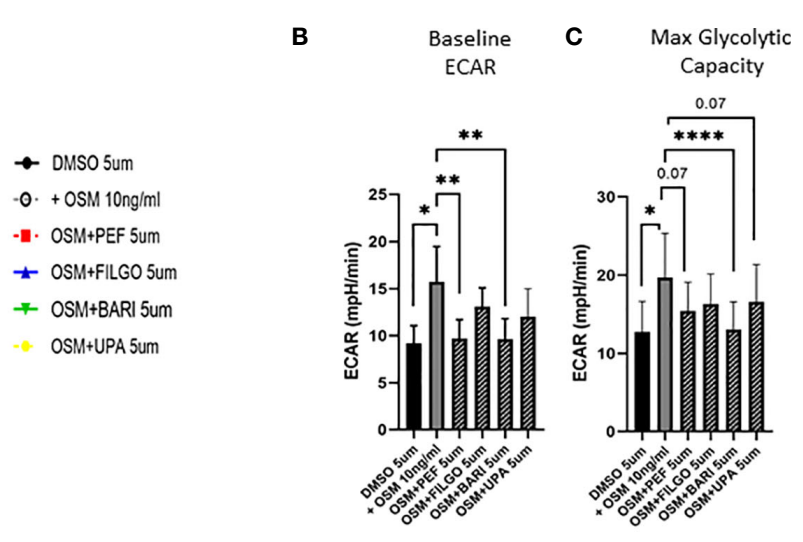

G

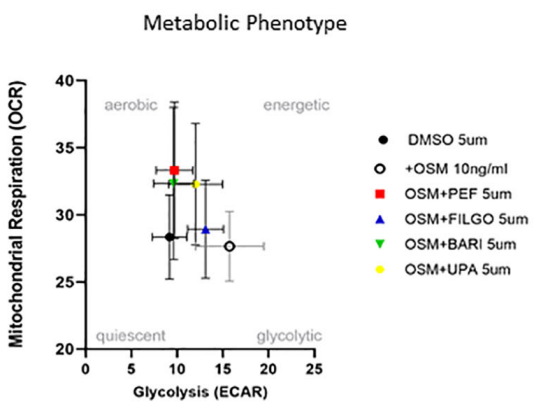

FIGURE 2 | JAKi reduce the increased glycolytic profile of PSAFLS induced by OSM. (A) PsAFLS were treated with JAKi (5 $\mu$ M) or DMSO (5 $\mu$ M) for $1 \mathrm{~h}$ and stimulated with OSM $(10 \mathrm{ng} / \mathrm{ml})$ for $24 \mathrm{~h}$. Average seahorse profiles demonstrating extracellular acidification rate (ECAR) (glycolysis) and oxygen consumption rate (OCR) (oxidative phosphorylation). (B) Representative bar graphs demonstrating baseline ECAR, (C) maximal glycolytic capacity, (D) baseline OCR, (E) maximal respiratory capacity, and (F) ECAR : OCR ratio. (G) Metabolic phenotype profile of PsAFLS, $n=6$. Values expressed as mean $+/-\mathrm{SEM},{ }^{*} p<0.05,{ }^{* *} p<0.01$,

${ }^{* \star \star \star} p<0.0001$ significantly different from DMSO+OSM control.

had the most significant reductions (Figure 5B). In parallel, we examined the effect of JAKi on the secretion of the cartilage destructive matrix metalloproteinase enzymes. Peficitinib significantly decreased MMP-1 ( $<<0.05)$, MMP-3 $(\mathrm{p}<0.05)$ and MMP-9 $(\mathrm{p}<0.05)$. While a decrease for all three MMPs was also observed in Upadacitinib, Filgotinib and Baricitinib treated cells, this did not reach significance (Figure 5C). In response to the OSM induced MMP-1 expression, gene analysis showed similar decreasing trends, with Peficitinib $(\mathrm{p}<0.01)$ and Baricitinib $(\mathrm{p}<0.05)$ displaying the greatest inhibition. Although not significant, Filgotinib and Upadacitinib both reduced MMP-1 expression (Figure 5D).

\section{JAK Inhibitors Block PsAFLS Migration Promoted by OSM}

Finally, the capacity of PsAFLS to migrate within the joint environment is associated with progressive and destructive joint disease, therefore, we next investigated the role of JAKi on migration of PsAFLS using a wound repair scratch assay. Figure 6A shows representative images demonstrating the increased migratory capacity of PsAFLS in response to OSM compared to unstimulated cells, in addition to the inhibitory effect of JAKi on PsAFLS migration, where repopulation of wound margins was inhibited by JAKi. Quantitative analysis demonstrated that OSM significantly induced migration of PsAFLS ( $p<0.01)$ compared to basal control (Figure 6B). However, Peficitinib $(\mathrm{p}<0.001)$, Filgotinib $(\mathrm{p}<0.01)$ and Baricitinib $(\mathrm{p}<0.05)$ all significantly decreased PsAFLS migration across the wound margins (Figure 6B). Although not significant, Upadacitinib also demonstrated strong inhibition of PsAFLS migration (Figure 6B). As migration of FLS is aided by adhesion molecules we also determined if JAKi influenced ICAM expression. Gene analysis showed a significant inhibition of OSM- induced ICAM expression by Upadacitinib $(\mathrm{p}<0.01)$, all other inhibitors displayed decreases in ICAM expression, although these did not reach significance (Figure 6C).

A summary table outlining the main effects of each JAKi can be found in Table 2 .

\section{DISCUSSION}

In this study, we identified the impact of four JAKi inhibitors; Peficitinib, Filgotinib, Baricitinib and Upadacitinib on the 
A

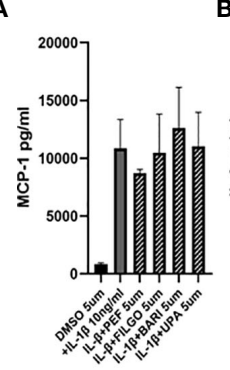

B

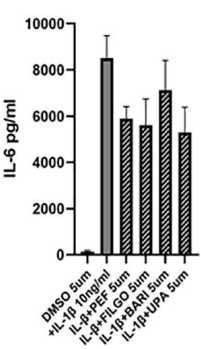

C

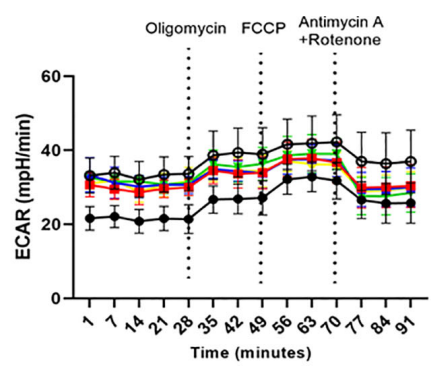

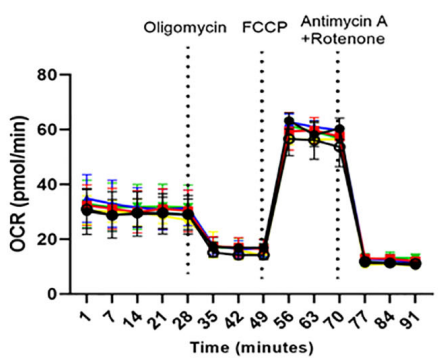

$\rightarrow$ DMSO 5 um

- +IL-1 $1 \beta 10 \mathrm{ng} / \mathrm{ml}$

- IL- $\beta+$ PEF 5UM

$\neq$ IL- $\beta+$ FILGO 5 um

- IL- $\beta$ +BARI 5um

IL-1 $\beta+$ UPA 5 um
D

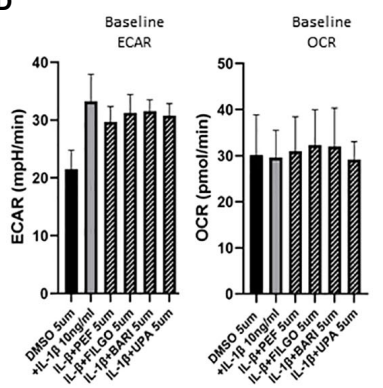

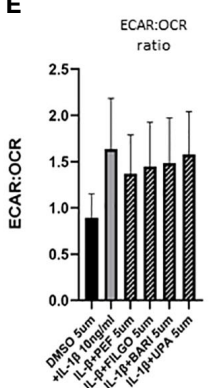

$\mathbf{F}$

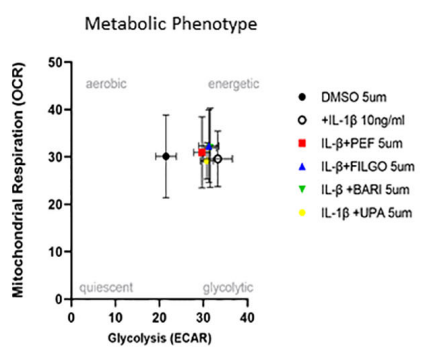

FIGURE 3 | Effect of JAKi on the IL-1ß-induced expression of pro-inflammatory mediators and bioenergetic profile in PsAFLS. (A, B) PSAFLS were treated with JAKi $(5 \mu \mathrm{M})$ or DMSO $(5 \mu \mathrm{M})$ for $1 \mathrm{~h}$ and stimulated with IL-1 $\beta(10 \mathrm{ng} / \mathrm{ml})$ for $24 \mathrm{~h}$. Bar graphs demonstrating secretion of (A) MCP-1 and (B) IL-6 by ELISA ( $\mathrm{n}=3)$. (C) Average seahorse profiles demonstrating extracellular acidification rate (ECAR) (glycolysis) and oxygen consumption rate (OCR) (oxidative phosphorylation). (D) Representative bar graphs demonstrating baseline ECAR and OCR and (E) ECAR : OCR ratio. (F) Metabolic phenotype profile in PSAFLS, $n=3$. Values expressed as mean +/- SEM.

A

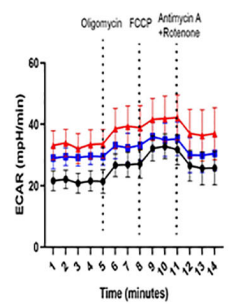

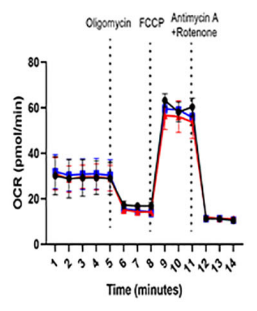

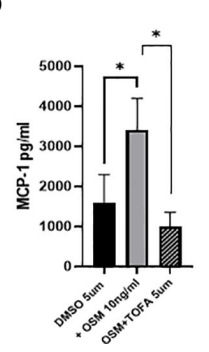

B

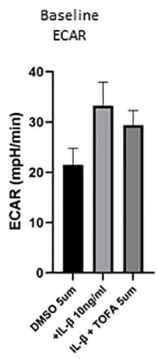

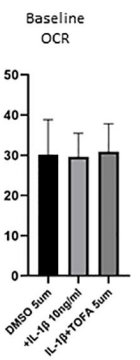

C

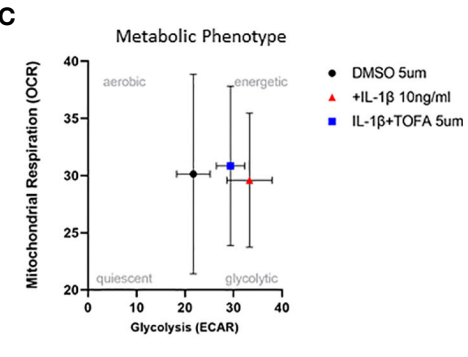

E
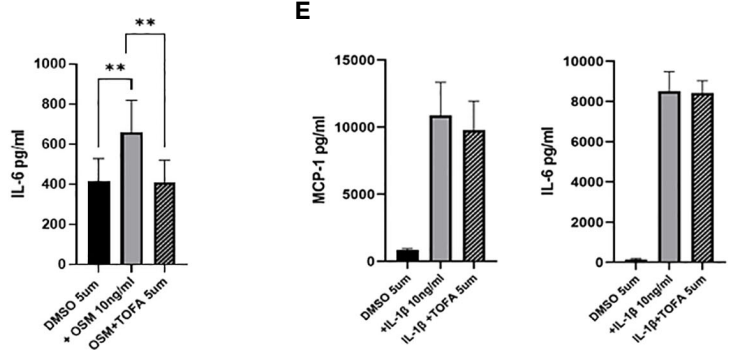

FIGURE 4 | Effect of Tofacitinib on the IL-1B or OSM induced expression of pro-inflammatory mediators and bioenergetic profile in PsAFLS. (A) Average seahorse profiles demonstrating extracellular acidification rate (ECAR) (glycolysis) and oxygen consumption rate (OCR) (oxidative phosphorylation). (B) Representative bar graphs demonstrating baseline ECAR and OCR. (C) Metabolic phenotype profile of PsAFLS, n=3. (D, E) PsAFLS were treated with Tofacitinib (5 $\mu$ M) or DMSO $(5 \mu \mathrm{M})$ for $1 \mathrm{~h}$ and stimulated with OSM (10ng/ml) $(\mathrm{n}=7)$ or IL-1 $\beta(10 \mathrm{ng} / \mathrm{ml})(\mathrm{n}=3)$ for $24 \mathrm{~h}$. and MCP-1 and IL-6 quantified. Values expressed as mean $+/-$ SEM ${ }^{*} p<0.05,{ }^{* *} p<0.01$. 
A

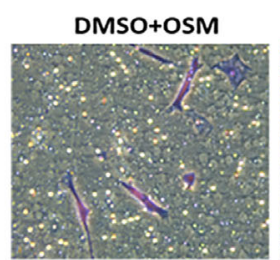

FILGO+OSM

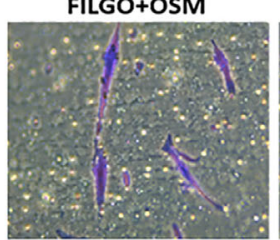

C

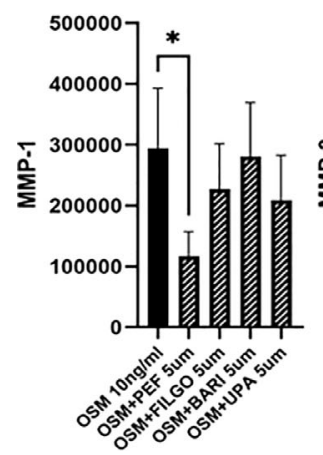

DMSO+OSM

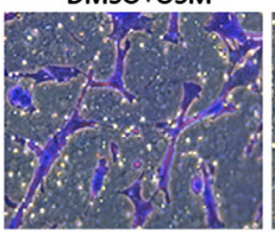

BARI+OSM
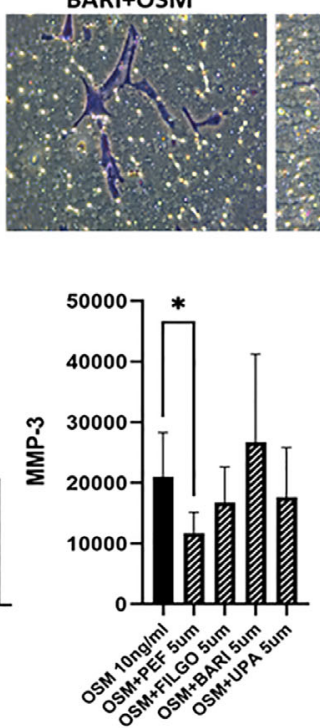

PEF+OSM

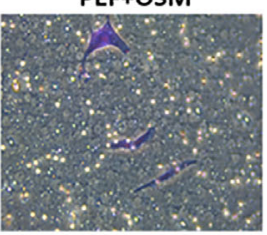

UPA+OSM
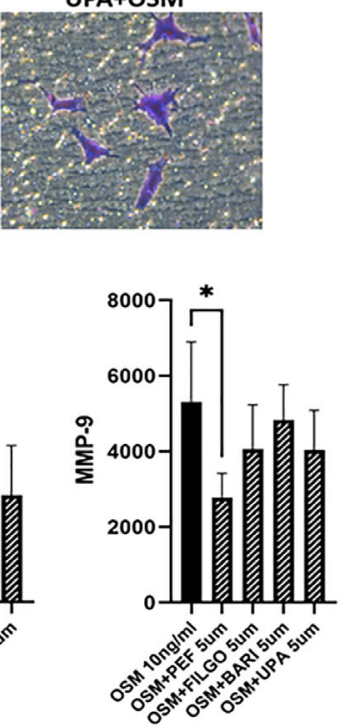

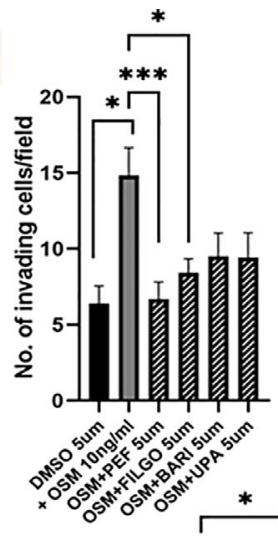

D

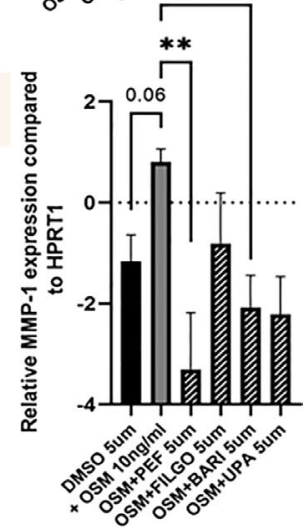

FIGURE 5 | OSM driven invasion by PSAFLS is blocked by JAKi. (A) Representative photomicrographs and (B) bar graph showing PSAFLS invasion following treatment with JAKi $(5 \mu \mathrm{M})$ or DMSO $(5 \mu \mathrm{M})$ for $1 \mathrm{hr}$ and stimulated with OSM (10 ng/ml) for $24 \mathrm{~h}, \mathrm{n}=6$ (magnification X20). (C) Representative bar graph showing MMP-1, MMP-3 and MMP-9 expression by MSD ELISA ( $n=7)$ and $(\mathbf{D})$ gene expression analysis of MMP-1 by real-time PCR $(n=5)$. Values expressed as the mean + - SEM, ${ }^{\star} p<0.05,{ }^{* *} p<0.01,{ }^{\star \star \star} p<0.001$ significantly different from DMSO+OSM control.

pathogenic phenotype observed in PsAFLS. JAKi are an encouraging class of drugs for the treatment of PsA, as evidence of increased JAK/STAT signalling has been shown at the site of inflammation $(15,21)$. To date, no study has examined the role of these JAKi on primary cells isolated from PsA synovial tissue. We utilised OSM as a stimulant, as it signals through the JAK-STAT pathway, specifically activating JAK1, JAK2 and to a lesser degree TYK2 (25). OSM is increased at the site of inflammation, in addition, several studies have shown that OSM drives synovial fibroblast invasive mechanisms (31-35). Using OSM to drive this inflammatory response, we demonstrate that JAKi significantly decreased the secretion of key pro-inflammatory mediators; MCP-1 and IL-6. This was accompanied by changes in the bioenergetics of the cells, whereby JAKi decreased the glycolytic profile of the PsAFLS resulting in a shift towards a more oxidative phosphorylated/quiescent phenotype. Finally, we demonstrated the ability of JAKi to inhibit the pathogenic function of PsAFLS by significantly decreasing their invasive and migratory capacity. While all JAKi inhibitors decreased proinflammatory and metabolic mechanisms in PsAFLS, this was most pronounced for Peficitinib. These data demonstrate that
JAK/STAT signalling mediates pro-inflammatory mechanisms that drive PsA pathogenesis, an effect inhibited with the use of JAKi.

In this study, we show that JAKi significantly reduce the secretion of OSM-induced pro-inflammatory mediators MCP-1 and IL-6, while displaying an increasing trend in IL-8 secretion, this differential regulation is consistent with the pleiotropic effects of OSM (36). We also show that Peficitinib, Baricitinib and Upadacitinib displayed the most significant inhibition of MCP-1 and IL-6 secretion, while Filgotinib also decreased cytokine secretion, this did not reach significance. The role for JAK-STAT signalling in PsA is consistent with studies showing increased expression of pSTAT3 and pSTAT1 in PsAFLS and PsA synovial tissue (21). Studies in psoriasis have shown an increase in pSTAT expression localised to the epidermal hyperproliferation layer $(37,38)$. Furthermore, the effect of JAKi is consistent with previous reports showing Tofacitinib, Peficitinib and Baricitinib inhibit IL-6 and MCP-1 expression in RAFLS (39, 40). As these JAKi can inhibit multiple pro-inflammatory mediators simultaneously and rescue function, they may act as a superior treatment for PsA compared to blockade of one specific cytokine. 


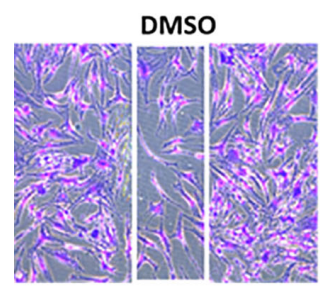

FILGO+OSM

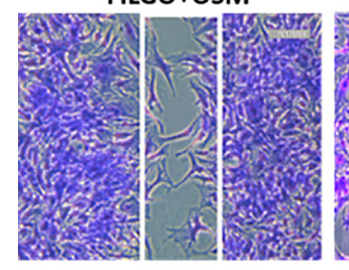

DMSO+OSM

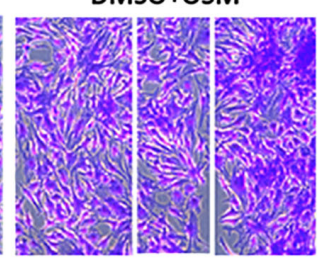

BARI+OSM

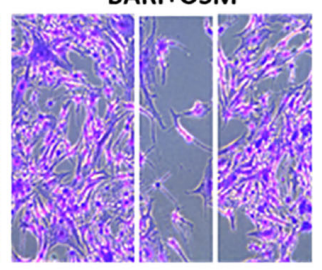

PEF+OSM

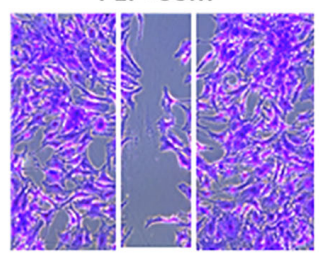

UPA+OSM

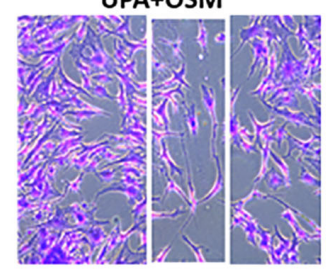

B

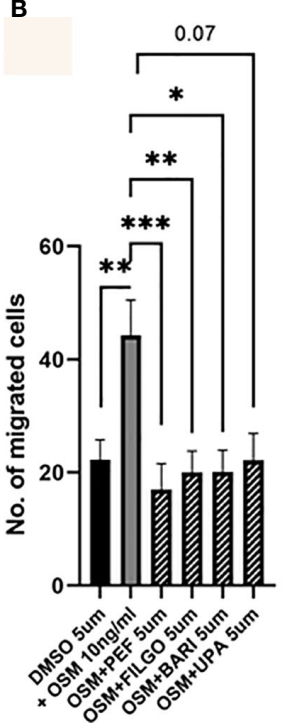

C

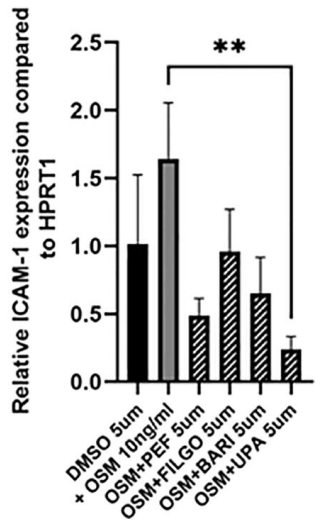

FIGURE 6 | JAKi inhibit OSM induced migration of PSAFLS. (A) Representative photomicrographs and (B) bar graph showing PsAFLS migration following treatment with JAKi $(5 \mu \mathrm{M})$ or DMSO $(5 \mu \mathrm{M})$ for $1 \mathrm{hr}$ and stimulated with OSM $(10 \mathrm{ng} / \mathrm{ml})$ for $24 \mathrm{~h}, \mathrm{n}=8$ (magnification X10). (C) Gene expression analysis of ICAM by real-time PCR $(n=5)$. Values expressed as mean $+/$ - SEM. ${ }^{*} p<0.05,{ }^{* *} p<0.01,{ }^{* \star *} p<0.001$ significantly different from DMSO+OSM control.

TABLE 2 | Summary of JAKi functions.

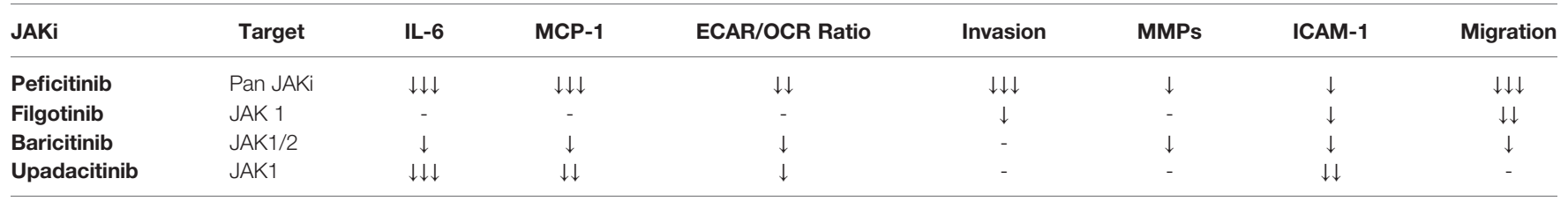

To examine if the effect of JAKi on pro-inflammatory function also alters the energy profile of PsAFLS, we investigated the two major energy pathways, glycolysis and oxidative phosphorylation using real-time Seahorse Technology. Changes in metabolism have been observed at the site of inflammation in both PsA and RA, due most likely to environmental factors within the joint resulting in a hypoxic microenvironment (41-44). Previous studies have shown a shift to a more glycolytic profile in RAFLS compared to OAFLS (45), with several studies demonstrating elevated levels of metabolic intermediates and increased activity of key glycolytic enzymes in both the RA and PsA synovium/cells (46-48). However, this is the first study to compare the bioenergetics of PsAFLS in response to treatment with JAKi. We show that JAKi reduced the glycolytic shift in favour of a more oxidative state, similar to a cell in quiescence. These findings are consistent with a report by McGarry et al. using Tofacitinib which inhibited glycolysis along with key glycolytic genes HK2, GSK3A, PDK1 and HIF1 $\alpha$ in RAFLS (39). Although all JAKi displayed inhibition, Peficitinib and Baricitinib significantly reduced the rates of glycolysis, with Peficitinib having the greatest effect on the ECAR/OCR ratio. Regulation of the metabolic pathways has been strongly linked with resolution of inflammation in the inflamed joint, with several studies demonstrating that metabolic blockade inhibits inflammation in vitro, ex vivo and in vivo models of arthritis (39, 42, 44, 45, 47-51).

Interestingly, interactions between JAK-STAT signalling and metabolic pathways have been demonstrated in previous studies. Blockade of the key glycolytic enzyme PFKFB3 inhibits pSTAT3 activation in RAFLS $(41,51,52)$. In turn, STAT3 itself can regulate glycolysis through HK2 in cancer cells $(41,51,52)$, and plays a key regulatory role in mediating interactions between HIF1 $\alpha$ and PKM2 (41, 51-54). Interplay between STAT3 and Sirtuin-1 has also been demonstrated to regulate oxygen consumption, ETC complex activity and metabolic intermediates in the mitochondria $(53,54)$. Indeed, studies have suggested that this effect may be due to localised STAT3 expression in the mitochondria which modulates the activity of complex I and II (52), however, other studies suggest alternative mechanisms, either via additional transcriptional regulation, or indirect activation of mitochondrial signalling pathways (55). In context of the inflamed joint, STAT3 interacts with various other key signalling pathways including HIFla, Notch and NFKB all of which regulate each other's activation through complex positive and negative feedback loops in the PsA/RA joint (56). Therefore, the use of JAKi in metabolically reprogramming these cells may aid in reducing their inflammatory aggressive phenotype in PsA. 
We also determined the effect of JAKi on PsAFLS function by examining their invasive and migratory capacity. All JAKi showed a striking inhibition of invasion by PsAFLS, although Peficitinib and Filgotinib displayed the strongest effect. In parallel, all JAKi significantly inhibited PsAFLS migration. While the precise mechanism by which JAKi impacts invasion and migration is unclear, Peficitinib significantly reduced MMP1, MMP-3, and MMP-9 secretion, while the other JAKi showed a slight decrease. Consistent with our data, several other studies using RAFLS have reported inhibition of MMP-1 and MMP-3 by both Tofacitinib and Peficitinib $(29,39,40)$, in addition to the inhibitory effect of Tofacitinib on PsAFLS invasion, network migration and migration (21). Furthermore, in PsA synovial explants, Tofacitinib inhibits MMP-3 expression and the overall MMP-3/TIMP ratio (21), thus reducing the ability of FLS to invade the tissue thereby reducing joint destruction. Other pathways involved in FLS invasion and migration include Integrin-cytoskeletal pathways that bridge cell-cell and cellECM interactions (57), with previous studies showing that JAK-STAT signalling regulates RA-FLS lamellipodia formation and RhoGTPases, key proteins involved in cellular movement (58). As migration of these cells to the joint is aided by adhesion molecules, we also show that ICAM expression is strongly reduced following treatment with JAKi. Similar effects were seen with RANKL, where Peficitinib and Tofacitinib decreased expression in RAFLS (34). Other potential mechanisms include the YAP pathway which has been implicated in RAFLS invasiveness $(59,60)$. Indeed, studies in fibroblasts from other disease settings have shown complex interactions between metabolic pathways and YAP/TAZ signalling (61). Therefore, the use of JAKi in PsA may help in reducing inflammation induced by infiltrating FLS to the inflamed joint.

Finally, in this study we utilised OSM as an activator of the JAK/STAT pathway, however, it is only one of many cytokines implicated in PsA pathogenesis that acts through this pathway, including IL-12, IL-23, IL-22 and IFN $\gamma$. Thus, there are limitations to the interpretation. While outside the scope of this study, an ideal model would be to culture PsAFLS with a cocktail of all the relevant cytokines that are known to be increased in the PsA joint in the presence or absence of JAKi.

In conclusion, this study demonstrates the effect of JAKi in targeting PsAFLS function in vitro, inhibiting invasive, migration and metabolic mechanisms leading to resolution of inflammation. These findings support the role for JAKi in patients with inadequate responses to current PsA therapies.

\section{REFERENCES}

1. Veale DJ, Fearon U. The Pathogenesis of Psoriatic Arthritis. Lancet (2018) 391(10136):2273-84. doi: 10.1016/S0140-6736(18)30830-4

2. Cafaro G, McInnes IB. Psoriatic Arthritis: Tissue-Directed Inflammation? Clin Rheumatol (2018) 37(4):859-68. doi: 10.1007/s10067-018-4012-7

3. van Kuijk AW, Tak PP. Synovitis in Psoriatic Arthritis: Immunohistochemistry, Comparisons With Rheumatoid Arthritis, and Effects of Therapy. Curr Rheumatol Rep (2011) 13(4):353-9. doi: 10.1007/s11926-011-0181-y

4. Ritchlin C, Haas-Smith SA, Hicks D, Cappuccio J, Osterland CK, Looney RJ. Patterns of Cytokine Production in Psoriatic Synovium. J Rheumatol (1998) 25:1544-52.

\section{DATA AVAILABILITY STATEMENT}

The raw data supporting the conclusions of this article will be made available by the authors, without undue reservation.

\section{ETHICS STATEMENT}

The studies involving human participants were reviewed and approved by St Vincent's University Hospital Research Ethics Committee. The patients/participants provided their written informed consent to participate in this study.

\section{AUTHOR CONTRIBUTIONS}

AO'B, MH, VM, SW, and KF performed the experiments, analysed the data, and prepared the manuscript. KF also processed clinical samples. UF conceived the experimental approach, analysed the data, and supervised and prepared the manuscript. DV conceived the experimental approach, collected the clinical samples, analysed the data, supervised the study, and prepared the manuscript. All authors contributed to the article and approved the submitted version.

\section{FUNDING}

This work was supported by the CARD Charity and Arthritis Ireland.

\section{ACKNOWLEDGMENTS}

The authors would like to acknowledge the financial support from Arthritis Ireland and the Centre for Arthritis and Rheumatic Disease, St. Vincent's University Hospital, UCD. We'd also like to thank the patients who were involved in the study.

5. Fromm S, Cunningham CC, Dunne MR, Veale DJ, Fearon U, Wade SM. Enhanced Angiogenic Function in Response to Fibroblasts From Psoriatic Arthritis Synovium Compared to Rheumatoid Arthritis. Arthritis Res Ther (2019) 21(1):297. doi: 10.1186/s13075-019-2088-3

6. Cañete JD, Rodríguez JR, Salvador G, Gómez-Centeno A, Muñoz-Gómez J, Sanmartí R. Diagnostic Usefulness of Synovial Vascular Morphology in Chronic Arthritis. A Systematic Survey of 100 Cases. Semin Arthritis Rheumatol (2003) 32(6):378-87. doi: 10.1053/sarh.2002.50004

7. Fearon U, Griosios K, Fraser A, Reece R, Emery P, Jones PF, et al. Angiopoietins, Growth Factors, and Vascular Morphology in Early Arthritis. J Rheumatol (2003) 30:260-8. 
8. Taams LS, Steel KJA, Srenathan U, Burns LA, Kirkham BW. IL-17 in the Immunopathogenesis of Spondyloarthritis. Nat Rev Rheumatol (2018) 14 (8):453-66. doi: 10.1038/s41584-018-0044-2

9. Wade SM, Canavan M, McGarry T, Low C, Wade SC, Mullan RH, et al. Association of Synovial Tissue Polyfunctional T-Cells With DAPSA in Psoriatic Arthritis. Ann Rheum Dis (2019) 78(3):350-4. doi: 10.1136/ annrheumdis-2018-214138

10. Steel KJA, Srenathan U, Ridley M, Durham LE, Wu SY, Ryan SE, et al. Polyfunctional, Proinflammatory, Tissue-Resident Memory Phenotype and Function of Synovial Interleukin-17A+CD8+ T Cells in Psoriatic Arthritis. Arthritis Rheumatol (2020) 72(3):435-47. doi: 10.1002/art.41156

11. Bridgewood C, Sharif K, Sherlock J, Watad A, McGonagle D. Interleukin-23 Pathway at the Enthesis: The Emerging Story of Enthesitis in Spondyloarthropathy. Immunol Rev (2020) 294(1):27-47. doi: 10.1111/imr.12840

12. Veale DJ, McGonagle D, McInnes IB, Krueger JG, Ritchlin CT, Elewaut D, et al. The Rationale for Janus Kinase Inhibitors for the Treatment of Spondyloarthritis. Rheumatol (Oxford) (2019) 58(2):197-205. doi: 10.1093/ rheumatology/key070

13. Darnell JE Jr. Stats and Gene Regulation. Science (1997) 277(5332):1630-5. doi: 10.1126/science.277.5332.1630

14. O'Shea JJ. Jaks, STATs, Cytokine Signal Transduction, and Immunoregulation: Are We There Yet? Immunity (1997) 7(1):1-11. doi: 10.1016/s1074-7613(00) 80505-1

15. Fiocco U, Accordi B, Martini V, Oliviero F, Facco M, Cabrelle A, et al. Jak/ Stat/Pkc $\delta$ Molecular Pathways in Synovial Fluid T Lymphocytes Reflect the THelper17 Expansion in Psa. Immunol Res (2014) 58:61-9. doi: 10.1007/ s12026-013-8481-0

16. Raychaudhuri SK, Abria C, Raychaudhuri SP. Regulatory Role of the JAK STAT Kinase Signalling System on the IL-23/IL-17 Cytokine Axis in Psoriatic Arthritis. Ann Rheum Dis (2017) 76(10):e36. doi: 10.1136/annrheumdis-2016211046

17. Marzaioli V, Canavan M, Floudas A, Wade SC, Low C, Veale DJ, et al. Monocyte-Derived Dendritic Cell Differentiation in Inflammatory Arthritis Is Regulated by the JAK/STAT Axis Via NADPH Oxidase Regulation. Front Immunol (2020) 11:1406. doi: 10.3389/fimmu.2020.01406

18. Kubo S, Yamaoka K, Kondo M, Yamagata K, Zhao J, Iwata S, et al. The JAK Inhibitor, Tofacitinib, Reduces the T Cell Stimulatory Capacity of Human Monocyte-Derived Dendritic Cells. Ann Rheum Dis (2014) 73(12):2192-8. doi: 10.1136/annrheumdis-2013-203756

19. De Wilde K, Martens A, Lambrecht S, Jacques P, Drennan MB, Debusschere K, et al. A20 Inhibition of STAT1 Expression in Myeloid Cells: A Novel Endogenous Regulatory Mechanism Preventing Development of Enthesitis. Ann Rheum Dis (2017) 76(3):585-92. doi: 10.1136/annrheumdis-2016-209454

20. Abji F, Pollock RA, Liang K, Chandran V, Gladman DD. Th17 Gene Expression in Psoriatic Arthritis Synovial Fluid and Peripheral Blood Compared to Osteoarthritis and Cutaneous Psoriasis. Clin Exp Rheumatol (2018) 36(3):486-9.

21. Gao W, McGarry T, Orr C, McCormick J, Veale DJ, Fearon U. Tofacitinib Regulates Synovial Inflammation in Psoriatic Arthritis, Inhibiting STAT Activation and Induction of Negative Feedback Inhibitors. Ann Rheum Dis (2016) 75(1):311-5. doi: 10.1136/annrheumdis-2014-207201

22. Ursini F, Russo E, De Giorgio R, De Sarro G, D'Angelo S. Current Treatment Options for Psoriatic Arthritis: Spotlight on Abatacept. Ther Clin Risk Manage (2018) 14:1053-9. doi: 10.2147/TCRM.S148586

23. Gadina M, Johnson C, Schwartz D, Bonelli M, Hasni S, Kanno Y, et al. Translational and Clinical Advances in JAK-STAT Biology: The Present and Future of Jakinibs. J Leukoc Biol (2018) 104(3):499-514. doi: 10.1002/ JLB.5RI0218-084R

24. Reinhardt A, Prinz I. Whodunit? The Contribution of Interleukin (Il)-17/Il22-Producing $\gamma \delta \mathrm{T}$ Cells, $\alpha \beta \mathrm{T}$ Cells, and Innate Lymphoid Cells to the Pathogenesis of Spondyloarthritis. Front Immunol (2018) 9:885. doi: 10.3389/ fimmu.2018.00885

25. Mease PJ, Lertratanakul A, Anderson JK, Papp K, Van den Bosch F, Tsuji S, et al. Upadacitinib for Psoriatic Arthritis Refractory to Biologics: SELECT-PsA 2. Ann Rheum Dis (2020) 80(3):312-20. doi: 10.1136/annrheumdis-2020-218870

26. Clinicaltrials.Gov. Identifier: Nct04115839 (Accessed 18/02/21).

27. Papp K, Pariser D, Catlin M, Wierz G, Ball G, Akinlade B, et al. A Phase 2a Randomized, Double-Blind, Placebo-Controlled, Sequential Dose-Escalation
Study to Evaluate the Efficacy and Safety of ASP015K, A Novel Janus Kinase Inhibitor, in Patients With Moderate-to-Severe Psoriasis. $\mathrm{Br} J$ Dermatol (2015) 173:767-76. doi: 10.1111/bjd.13745

28. Papp KA, Menter MA, Raman M, Disch D, Schlichting DE, Gaich C, et al. A Randomized Phase 2b Trial of Baricitinib, An Oral Janus Kinase (JAK) 1/ JAK2 Inhibitor, in Patients With Moderate-To-Severe Psoriasis. Br J Dermatol (2016) 174(6):1266-76. doi: 10.1111/bjd.14403

29. Diller M, Hasseli R, Hülser ML, Aykara I, Frommer K, Rehart S, et al. Targeting Activated Synovial Fibroblasts in Rheumatoid Arthritis by Peficitinib. Front Immunol (2019) 10:541. doi: 10.3389/fimmu.2019.00541

30. Le Goff B, Singbrant S, Tonkin BA, Martin TJ, Romas E, Sims NA, et al. Oncostatin M Acting Via OSMR, Augments the Actions of IL-1 and TNF in Synovial Fibroblasts. Cytokine (2014) 68(2):101-9. doi: 10.1016/j.cyto.2014.04.001

31. Choy EH. Clinical Significance of Janus Kinase Inhibitor Selectivity. Rheumatol (Oxford) (2019) 58(6):953-62. doi: 10.1093/rheumatology/key339

32. Manicourt DH, Poilvache P, Van Egeren A, Devogelaer JP, Lenz ME, Thonar EJ. Synovial Fluid Levels of Tumor Necrosis Factor Alpha and Oncostatin M Correlate With Levels of Markers of the Degradation of Crosslinked Collagen and Cartilage Aggrecan in Rheumatoid Arthritis But Not in Osteoarthritis. Arthritis Rheumatol (2000) 43(2):281-8. doi: 10.1002/1529-0131(200002) 43:2<281::AID-ANR7>3.0.CO;2-7

33. Fearon U, Reece R, Smith J, Emery P, Veale DJ. Synovial Cytokine and Growth Factor Regulation of MMPs/TIMPs: Implications for Erosions and Angiogenesis in Early Rheumatoid and Psoriatic Arthritis Patients. Ann N Y Acad Sci (1999) 878:619-21. doi: 10.1111/j.1749-6632.1999.tb07743.x

34. Fearon U, Mullan R, Markham T, Connolly M, Sullivan S, Poole AR, et al. Oncostatin M Induces Angiogenesis and Cartilage Degradation in Rheumatoid Arthritis Synovial Tissue and Human Cartilage Cocultures. Arthritis Rheumatol (2006) 54(10):3152-62. doi: 10.1002/art.22161

35. Langdon C, Leith J, Smith F, Richards CD. Oncostatin M Stimulates Monocyte Chemoattractant Protein-1- and Interleukin-1-Induced Matrix Metalloproteinase-1 Production by Human Synovial Fibroblasts In Vitro. Arthritis Rheumatol (1997) 40(12):2139-46. doi: 10.1002/art.1780401207

36. Hanlon MM, Rakovich T, Cunningham CC, Ansboro S, Veale DJ, Fearon U, et al. Stat3 Mediates the Differential Effects of Oncostatin M and Tnf $\alpha$ on RA Synovial Fibroblast and Endothelial Cell Function. Front Immunol (2019) 10:2056. doi: 10.3389/fimmu.2019.02056

37. Hald A, Andrés RM, Salskov-Iversen ML, Kjellerup RB, Iversen L, Johansen C. STAT1 Expression and Activation Is Increased in Lesional Psoriatic Skin. Br J Dermatol (2013) 168(2):302-10. doi: 10.1111/bjd.12049

38. Honma M, Fujii M, Iinuma S, Minami-Hori M, Takahashi H, IshidaYamamoto A, et al. Podoplanin Expression in Wound and Hyperproliferative Psoriatic Epidermis: Regulation by Tgf $\beta$ and STAT3 Activating Cytokines, Ifn $\gamma$, IL6, and IL22. J Dermatol Sci (2012) 65:134-40. doi: 10.1016/j.jdermsci.2011.11.011

39. McGarry T, Orr C, Wade S, Biniecka M, Wade S, Gallagher L, et al. Jak/Stat Blockade Alters Synovial Bioenergetics, Mitochondrial Function, and Proinflammatory Mediators in Rheumatoid Arthritis. Arthritis Rheumatol (2018) 70(12):1959-70. doi: 10.1002/art.40569

40. Emori T, Kasahara M, Sugahara S, Hashimoto M, Ito H, Narumiya S, et al. Role of JAK-STAT Signaling in the Pathogenic Behavior of Fibroblast-Like Synoviocytes in Rheumatoid Arthritis: Effect owf the Novel JAK Inhibitor Peficitinib. Eur J Pharmacol (2020) 882:173238. doi: 10.1016/j.ejphar.2020.173238

41. Biniecka M, Canavan M, McGarry T, Gao W, McCormick J, Cregan S, et al. Dysregulated Bioenergetics: A Key Regulator of Joint Inflammation. Ann Rheum Dis (2016) 75(12):2192-200. doi: 10.1136/annrheumdis-2015-208476

42. Ng CT, Biniecka M, Kennedy A, McCormick J, Fitzgerald O, Bresnihan B, et al. Synovial Tissue Hypoxia and Inflammation In Vivo. Ann Rheum Dis (2010) 69(7):1389-95. doi: 10.1136/ard.2009.119776

43. Haas R, Smith J, Rocher-Ros V, Nadkarni S, Montero-Melendez T, D'Acquisto F, et al. Lactate Regulates Metabolic and Pro-Inflammatory Circuits in Control of T Cell Migration and Effector Functions. PloS Biol (2015) 13(7):e1002202. doi: 10.1371/journal.pbio.1002202

44. Shen Y, Wen Z, Li Y, Hong J, Goronzy JJ, Weyand CM, et al. Metabolic Control of the Scaffold Protein TKS5 in Tissue-Invasive, Proinflammatory T Cells. Nat Immunol (2017) 18(9):1025-34. doi: 10.1038/ni.3808

45. Ahn JK, Kim S, Hwang J, Kim J, Kim KH, Cha HS. Gc/Tof-MS-Based Metabolomic Profiling in Cultured Fibroblast-Like Synoviocytes From 
Rheumatoid Arthritis. Joint Bone Spine (2016) 83(6):707-13. doi: 10.1016/ j.jbspin.2015.11.009

46. Fearon U, Hanlon MM, Wade SM, Fletcher JM. Altered Metabolic Pathways Regulate Synovial Inflammation in Rheumatoid Arthritis. Clin Exp Immunol (2019) 197(2):170-80. doi: 10.1111/cei.13228

47. Hua S, Dias TH. Hypoxia-Inducible Factor (HIF) as a Target for Novel Therapies in Rheumatoid Arthritis. Front Pharmacol (2016) 7:184. doi: 10.3389/fphar.2016.00184

48. Bustamante MF, Oliveira PG, Garcia-Carbonell R, Croft AP, Smith JM, Serrano RL, et al. Hexokinase 2 as a Novel Selective Metabolic Target for Rheumatoid Arthritis. Ann Rheum Dis (2018) 77(11):1636-43. doi: 10.1136/ annrheumdis-2018-213103

49. Garcia-Carbonell R, Divakaruni AS, Lodi A, Vicente-Suarez I, Saha A, Cheroutre $\mathrm{H}$, et al. Critical Role of Glucose Metabolism in Rheumatoid Arthritis Fibroblast-Like Synoviocytes. Arthritis Rheumatol (2016) 68 (7):1614-26. doi: 10.1002/art.39608

50. Pucino V, Certo M, Bulusu V, Cucchi D, Goldmann K, Pontarini E, et al. Lactate Buildup at the Site of Chronic Inflammation Promotes Disease by Inducing $\mathrm{CD}^{+} \mathrm{T}$ Cell Metabolic Rewiring. Cell Metab (2019) 30(6):10551074.e8. doi: 10.1016/j.cmet.2019.10.004

51. Li M, Jin R, Wang W, Zhang T, Sang J, Li N, et al. STAT3 Regulates Glycolysis Via Targeting Hexokinase 2 in Hepatocellular Carcinoma Cells. Oncotarget (2017) 8(15):24777-84. doi: 10.18632/oncotarget.15801

52. Shi LZ, Wang R, Huang G, Vogel P, Neale G, Green DR, et al. HIFlalphaDependent Glycolytic Pathway Orchestrates a Metabolic Checkpoint for the Differentiation of TH17 and Treg Cells. J Exp Med (2011) 208(7):1367-76. doi: 10.1084/jem.20110278

53. Gao X, Wang H, Yang JJ, Chen J, Jie J, Li L, et al. Reciprocal Regulation of Protein Kinase and Pyruvate Kinase Activities of Pyruvate Kinase M2 by Growth Signals. J Biol Chem (2013) 288(22):15971-9. doi: 10.1074/jbc.M112.448753

54. Bernier M, Paul RK, Martin-Montalvo A, Scheibye-Knudsen M, Song S, $\mathrm{He} \mathrm{HJ}$, et al. Negative Regulation of STAT3 Protein-Mediated Cellular Respiration by SIRT1 Protein. J Biol Chem (2011) 286(22):19270-9. doi: 10.1074/jbc.M110.200311

55. Phillips D, Reilley MJ, Aponte AM, Wang G, Boja E, Gucek M, et al. Stoichiometry of STAT3 and Mitochondrial Proteins: Implications for the
Regulation of Oxidative Phosphorylation by Protein-Protein Interactions. J Biol Chem (2010) 285(31):23532-6. doi: 10.1074/jbc.C110.152652

56. Gao W, McCormick J, Connolly M, Balogh E, Veale DJ, Fearon U. Hypoxia and STAT3 Signalling Interactions Regulate Pro-Inflammatory Pathways in Rheumatoid Arthritis. Ann Rheum Dis (2015) 74(6):1275-83. doi: 10.1136/ annrheumdis-2013-204105

57. McGarry T, Veale DJ, Gao W, Orr C, Fearon U, Connolly M. Toll-Like Receptor 2 (TLR2) Induces Migration and Invasive Mechanisms in Rheumatoid Arthritis. Arthritis Res Ther (2015) 17(1):153. doi: 10.1186/ s13075-015-0664-8

58. Lao M, Shi M, Zou Y, Huang M, Ye Y, Qiu Q, et al. Protein Inhibitor of Activated Stat3 Regulates Migration, Invasion, and Activation of FibroblastLike Synoviocytes in Rheumatoid Arthritis. J Immunol (2016) 196(2):596606. doi: 10.4049/jimmunol.1403254

59. Bottini A, Wu DJ, Ai R, Le Roux M, Bartok B, Bombardieri M, et al. PTPN14 Phosphatase and YAP Promote Tgf $\beta$ Signalling in Rheumatoid Synoviocytes. Ann Rheum Dis (2019) 78(5):600-9. doi: 10.1136/annrheumdis-2018-213799

60. Zhou W, Shen Q, Wang H, Yang J, Zhang C, Deng Z, et al. Knockdown of YAP/TAZ Inhibits the Migration and Invasion of Fibroblast Synovial Cells in Rheumatoid Arthritis by Regulating Autophagy. J Immunol Res (2020) 2020:9510594. doi: 10.1155/2020/9510594

61. Gui Y, Li J, Lu Q, Feng Y, Wang M, He W, et al. Yap/Taz Mediates mTORC2Stimulated Fibroblast Activation and Kidney Fibrosis. J Biol Chem (2018) 293 (42):16364-75. doi: 10.1074/jbc.RA118.004073

Conflict of Interest: The authors declare that the research was conducted in the absence of any commercial or financial relationships that could be construed as a potential conflict of interest.

Copyright (๑) 2021 O'Brien, Hanlon, Marzaioli, Wade, Flynn, Fearon and Veale. This is an open-access article distributed under the terms of the Creative Commons Attribution License (CC BY). The use, distribution or reproduction in other forums is permitted, provided the original author $(s)$ and the copyright owner(s) are credited and that the original publication in this journal is cited, in accordance with accepted academic practice. No use, distribution or reproduction is permitted which does not comply with these terms. 